\title{
CARACTERÍSTICAS DA CARCAÇA E DA CARNE DE CORDEIROS CORRIEDALE MANEJADOS EM DUAS ALTURAS DE MILHETO
}

\author{
CARCASS AND MEAT CHARACTERISTICS FROM CORRIEDALE LAMBS GRAZING TWO \\ HEIGHTS OF PEARL MILLET
}

\author{
Lemes, J.S. ${ }^{1 A} ;$ Osório, M.T.M. ${ }^{2 A}$; Osório, J.C.S. ${ }^{2 B} ;$ Gonzaga, S.S. ${ }^{3} ;$ Martins, L.S. ${ }^{1 B}$; \\ Esteves, R.M.G. ${ }^{2 C}$ e Lehmen, R.I. ${ }^{4}$
}

1PPGZ/UFPel. Brasil. Aschneiderlemes@yahoo.com.br; Bvipmartins@yahoo.com.br

${ }^{2}$ Faculdade de Ciências Agrárias. UFGD. Dourados, MT. Brasil. Amtosorio@ufpel.tche.br; Bjcosorio@pq.cnpq.br; 'esteves@ufpel.tche.br

${ }^{3}$ EMBRAPA-CPPSUL. Bagé, RS. Brasil. gonzaga@cppsul.embrapa.br

${ }^{4}$ Agronomia/UPF. Brasil. rosileneil@yahoo.com.br

PalaVRas ChaVe adicionais

Composição tecidual. Pennisetum americanum.

\section{RESUMO}

O objetivo deste estudo foi avaliar características de qualidade da carcaça e da carne de cordeiros Corriedale manejados em duas alturas de pastejo em milheto. A terminação dos cordeiros foi feita em pastagem anual de verão, milheto cultivar comum (Pennisetum americanum (L.) Leeke.). Os tratamentos consistiram de duas alturas de pastejo, 20 e $40 \mathrm{~cm}$. Os animais foram abatidos baseando-se na condição corporal ao atingirem o índice entre 2 e 3 (1 a 5). Foram realizadas avaliações quantitativas e qualitativas da carcaça e da carne. A conformação apresentou alta relação com o estado de engorduramento. Não houve diferença entre as características subjetivas de textura e cor, já para o marmoreio houve efeito da altura do milheto $(p<0,01)$, onde cordeiros que pastejaram em potreiros com $20 \mathrm{~cm}$ de altura de milheto obtiveram maior índice. Concluise que não há diferença entre as características da carcaça, instrumentais da carne e teciduais da paleta de cordeiros Corriedale manejados em diferentes alturas de pastejo de milheto, quanto às características subjetivas, a altura do milheto influencia o índice de marmoreio na carne.

\section{SUMMARY}

The objective of this study was to evaluate the carcass characteristics and meat quality of

\section{AdDitional KEYWORDS}

Pennisetum americanum. Tissue composition.

Corriedale lambs subjected to two grazing height on millet. The lamb's termination was done in summer annual pasture on a common cultivar of millet (Pennisetum americanum (L.) Leeke). The treatments consisted of two grazing heights, 20 and $40 \mathrm{~cm}$. The slaughter was based on body condition between 2 and 3 ( 1 to 5 scale). Quantitative and qualitative evaluations were carried out on carcass and meat. The conformation demonstrated great relation with fattening status. There was no difference between subjective characteristics of color and texture. However marbling index values were higher $(p<0.01)$, when lambs grazed on $20 \mathrm{~cm}$ height millet. In conclusion, there is neither difference between carcass characteristics and instrumental meat evaluations, nor difference in tissue components of the shoulder in Corriedale lambs subjected to different grazing heights on millet. However, the height of grazing affects the marbling score.

\section{INTRODUÇÃO}

Na ovinocultura gaúcha, a carne, principalmente a de cordeiro, passou a ser uma alternativa para incremento da economia do setor. Contudo, tanto no Rio Grande do Sul, como em outros Estados brasileiros, vem 


\section{LEMES, OSÓRIO, OSÓRIO, GONZAGA, MARTINS, ESTEVESE LEHMEN}

crescendo os estudos sobre a qualidade da carcaça e da carne, especialmente desta última e, dos fatores que atuam sobre referida qualidade para que se possa otimizar a produção e oferecer subsídios para o entendimento da cadeia produtiva e uma justa comercialização. No entanto, ainda faltam conhecimentos sobre a adequação alimentar, principalmente, das pastagens, nos sistemas extensivos e/ou semi-extensivos e a comercialização de ovinos para o abate que ainda é realizada com referência ao peso vivo e/ou rendimento de carcaça fria, sem que a qualidade do produto seja considerada pelas casas de carne e frigoríficos.

O valor econômico de uma carcaça ovina depende essencialmente do peso, conformação, proporção dos principais tecidos (músculo, gordura e osso), sua distribuição na carcaça, grossura do músculo e qualidade da carne (Jardim et al., 2000). Assim, a valorização da carcaça está diretamente relacionada à sua porção comestível, que deve ser aquela que apresente a máxima quantidade de músculo, com adequada quantidade de gordura, ou seja, a relação músculo/gordura que provoque o mais alto grau de satisfação ao consumidor (Osório et al., 2002). Portanto, a relação entre medidas in vivo e da carcaça é de suma importância para o melhoramento genético e no entendimento comercial da cadeia da carne (Bonacina et al., 2007a).

Sendo assim, o conhecimento da composição tecidual dos cortes da carcaça de ovinos é de grande importância, pois visa a melhorar os aspectos qualitativos e quantitativos dos cortes, facilitando a comercialização através da obtenção de produtos que propiciam maior grau de satisfação do consumidor. A paleta é um corte com adequada predição tecidual da carcaça, pois apresenta altos coeficientes de correlação com a composição total da mesma (Oliveira et al., 1998).

Entre as características que determinam a qualidade da carcaça e da carne ovina podemos destacar a cor, maciez e capacidade de retenção de água, que consiste na habilidade de retenção de água durante a aplicação de força ou tratamentos externos. Já a avaliação instrumental da carne permite conhecer a preferência do mercado consumidor e, conseqüentemente, fixar a qualidade do produto que propicia maior grau de satisfação. Inúmeras estratégias têm sido utilizadas para conseguir atender a demanda dos consumidores por carne de maior qualidade e, com isso, são necessárias pesquisas que avaliam as características do produto final e as relações dessas com as preferências dos compradores.

No entanto, um dos grandes gargalos para o avanço da ovinocultura no Rio Grande do Sul é o complexo inanição/exposição ao ambiente, sendo a principal causa de mortalidade perinatal de cordeiros (Oliveira et al., 1995). Apesar de sua sobrevivência em locais degradados, ou com menor disponibilidade forrageira, produzem bem abaixo do seu potencial. Logo, buscar a terminação dos ovinos com alimentos mais eficientes vem sendo solicitado pelos produtores. A eficiência do processo de produção de carne ovina depende do desempenho reprodutivo das ovelhas, tendo como elemento central o cordeiro, cuja velocidade de crescimento é determinada basicamente pela qualidade do alimento ingerido. Desse modo, a alimentação é um fator determinante no crescimento dos cordeiros e da qualidade de sua carcaça e carne, ainda que apresentem marcadas diferenças em função das características do sistema de produção (Jardim et al., 2000).

Entre as espécies de forrageiras anuais cultivadas no Rio Grande do Sul, o milheto (Pennisetum americanum L. Leeke) é a gramínea de estação quente mais utilizada para pastejo. Basicamente por suas características agronômicas de alta resistência à seca, adaptação a solos de fertilidade moderada, crescimento rápido, boa produção de forragem, aliado ainda à qualidade forrageira, bem como pelo amplo período de 
utilização (Durães et al., 2003).

Dessa forma, o objetivo deste estudo foi avaliar características que visam à qualidade da carcaça e da carne de cordeiros Corriedale manejados em duas alturas de pastejo em milheto.

\section{MATERIALEMÉTODOS}

O trabalho foi realizado no Centro de Pesquisas de Pecuária dos Campos Sul Brasileiros (EMBRAPA- CPPSUL), Bagé, RS, na fase de campo e no Departamento de Zootecnia, FAEM / UFPel, Pelotas, RS, na fase laboratorial, utilizando-se 32 cordeiros machos, não-castrados, da raça Corriedale.

A terminação dos cordeiros foi feita em dois piquetes em pastagem anual de verão, milheto cv. comum; (Pennisetum americanum (L.) Leeke.) com área total de 1,5 hectare. Os tratamentos consistiram de duas alturas de condução de pastejo, 20 (18 animais) com disponibilidade de forragem de $907 \mathrm{~kg}$ de MS/há e 40 cm (14 animais), com $1442 \mathrm{~kg}$ de MS/ha. Os animais foram vacinados e desverminados na entrada na pastagem de milheto. O controle de doenças infecto-contagiosas e parasitárias obedeceu aos critérios preconizados pela equipe de sanidade animal da Embrapa Pecuária Sul.

Os animais foram abatidos baseando-se na condição corporal ao atingirem o índice entre 2 e 3, numa escala de 1 a 5 (Osório e Osório, 2005); por ser esse o índice de condição corporal considerado adequado a preferência do consumidor segundo o Conselho Regulador Herval Premium.

No momento do abate os cordeiros estavam com idade em torno de 140 dias. Antes do abate realizaram-se as seguintes avaliações (Osório e Osório, 2005): peso corporal (kg), conformação - avaliação visual - portanto subjetiva, considerando o desenvolvimento muscular do animal como um todo ( $1=$ muito pobre a $5=$ excelente), condição corporal, medida subjetiva realizada para avaliar o estado de gordura e a relação entre as diferentes partes do animal
(1,0= menor até $5,0=$ maior $)$, comprimento corporal, distância compreendida entre a articulação escapulo-umeral até a ponta da nádega (cm), compacidade corporal medida do peso corporal ao abate divide pelo comprimento corporal, que resultaria em kg/ cm (PV/CC). Após, concluídas as avaliações in vivo, realizou-se o abate dos animais através da sangria por meio de corte na artéria carótida e veias jugulares, posteriormente efetuou-se a esfola, evisceração e retirada da cabeça e das patas. Os componentes corporais foram pesados imediatamente.

A avaliação das carcaças foi realizada na tarde do sacrifício, após permanência da carcaça por quatro horas em resfriamento a temperatura ambiente, quando foram procedidas as avaliações e medidas descritivas da carcaça (Osório e Osório, 2005). As medidas obtidas foram peso da carcaça (kg), conformação e compacidade da carcaça, estado de engorduramento que é a avaliação visual da quantidade e distribuição da gordura, não somente da gordura de cobertura, mas, também, a gordura renal e pélvica ( $1=$ muito pobre a $5=$ excelente). Após foi realizada a separação regional dos cortes comerciais e pesadas as seguintes peças: perna, paleta, peito e costelas fixas e flutuantes.

Para realizar as análises da qualidade da carne foi medido no músculo Longissimus dorsi (lombo), entre a $12^{\mathrm{a}}$ e $13^{\mathrm{a}}$ costela, e o músculo tríceps braquial (paleta), o pH zero hora, com eletrodo de penetração. Após as carcaças foram acondicionadas em câmara fria, com ar forçado, a temperatura de $4{ }^{\circ} \mathrm{C}$ por 24 horas, quando foi medido novamente o pH dos músculos.

Após 24 horas do abate foi realizada a avaliação visual, onde foram determinadas as características subjetivas da carne: textura (1,0= muito áspera até $5,0=$ muito fina), marmoreio ( $1=0$ não existente até $5,0=$ excessivo) e cor $(1,0=$ rosa claro até $5,0=$ vermelho escuro). 
Na análise instrumental foi utilizado para avaliação da capacidade de retenção de água, o método de pressão (Sierra, 1973) e para cor, utilizando colorimetro Minolta Chroma Meter CR-300 (sistema CIE L*a*b*) e pelo físico-químico de Hornsey (1956) ou ferro hemínico, por transmitância, todas realizadas 48 horas após o abate, os músculos foram mantidos sob refrigeração de 1 a $4{ }^{\circ} \mathrm{C}$. Já para as medidas de composição tecidual, realizadas na paleta, a qual compreende parte do membro anterior, incluindo a musculatura da escápula e na parte distal a secção é feita ao nível da porção média dos ossos do carpo e o peso é tomado em quilogramas. Os cortes foram identificados e armazenados em sacos de plástico e acondicionados em freezer a $-18^{\circ} \mathrm{C}$. Após o descongelamento das peças, em geladeira a $10^{\circ} \mathrm{C}$, por 24 horas, dentro dos sacos de plástico, retiraram-se estes e foram pesadas cada peça individualmente. Realizou-se a dissecação das paletas com auxílio de bisturi, para determinação da composição tecidual em gorduras (gordura subcutânea e intermuscular), músculos (total de músculos dissecados, após a remoção completa de todas as gorduras subcutânea e intermuscular aderidas), ossos (dissecados após a remoção completa de todo o músculo e gorduras subcutânea e intermuscular aderidas) e tecidos considerados outros (fáscias, tendões, linfonodo e grandes vasos). Os componentes dos teciduais foram pesados individualmente para serem expressos em relação ao peso total da paleta.

Os dados foram submetidos à ANOVA do programa NCSS 7.0(2005), com o seguinte modelo estatístico:

Tabela I. Médias e erros padrão das características in vivo e na carcaça em cordeiros da raça Corriedale manejados em duas diferentes alturas de milheto. (Means and standard errors of the in vivo and carcass characteristics of Corriedale lambs grazing millet to two heights).

\begin{tabular}{lccc}
\hline Altura do milheto & $\begin{array}{c}20 \mathrm{~cm} \\
\mathrm{n}=18\end{array}$ & $\begin{array}{l}40 \mathrm{~cm} \\
\mathrm{n}=14\end{array}$ & $\mathrm{p}$ \\
\hline In vivo & & & \\
Peso (kg) & $31,37 \pm 0,33$ & $30,92 \pm 038$ & 0,3875 \\
Conformação (1-5) & $3,36 \pm 0,09$ & $3,07 \pm 0,10$ & 0,0385 \\
Condição corporal (1-5) & $2,26 \pm 0,07$ & $2,23 \pm 0,08$ & 1,0000 \\
Comprimento corporal (cm) & $60,24 \pm 0,01$ & $57,54 \pm 0,02$ & 0,1097 \\
Compacidade corporal (kg/cm) & $0,52 \pm 0,01$ & $0,54 \pm 0,01$ & 0,3007 \\
\hline Carcaça & & & \\
Peso (kg) & $11,50 \pm 0,36$ & $11,90 \pm 0,41$ & 0,4726 \\
Conformação (1-5) & $3,23 \pm 0,13$ & $3,13 \pm 0,15$ & 0,5643 \\
Comprimento da carcaça (cm) & $58,75 \pm 0,38$ & $58,04 \pm 0,45$ & 0,2359 \\
Perna (kg) & $2,137 \pm 0,072$ & $2,097 \pm 0,082$ & 0,5052 \\
Paleta (kg) & $1,285 \pm 0,039$ & $1,271 \pm 0,044$ & 0,5212 \\
Peito (kg) & $0,657 \pm 0,027$ & $0,658 \pm 0,031$ & 0,9993 \\
Costelas fixas (kg) & $0,942 \pm 0,045$ & $0,894 \pm 0,051$ & 0,1622 \\
Costelas flutuantes (kg) & $0,380 \pm 0,019$ & $0,397+0,021$ & 0,5018 \\
Espessura da gordura de cobertura (mm) & $1,06 \pm 0,06$ & $1,06 \pm 0,07$ & 0,9760 \\
Estado de engorduramento (1-5) & $2,51 \pm 0,12$ & $2,49 \pm 0,14$ & 0,9417 \\
Rendimento de carcaça (\%) & $37,16 \pm 0,55$ & $37,69 \pm 0,62$ & 0,4162 \\
Compacidade da carcaça (kg/cm) & $0,198 \pm 0,005$ & $0,200 \pm 0,006$ & 0,2193 \\
& & & \\
\hline
\end{tabular}

Archivos de zootecnia vol. 63, núm. 241, p. 164. 


$$
Y_{i j k}=\mu+E_{i}+e_{i j k}
$$

onde:

$Y_{\mathrm{ijk}}=$ observação de peso de um componente tecidual da paleta de cordeiro ou valor de uma característica in vivo e da carcaça ou de qualidade da carne;

$\mu=$ média geral;

$E_{i}=$ efeito da altura de pastejo em milheto; $\mathrm{e}_{\mathrm{ijk}}=$ erro experimental.

O peso da carcaça foi utilizado como covariável no modelo.

As análises de correlação e regressão linear entre as características in vivo e na carcaça também foram realizadas através do programa NCSS 7.0 (2005).

\section{RESULTADOSEDISCUSSÃO}

Nas características morfológicas in vivo e na carcaça, a análise de variância demonstrou que não há diferença $(\mathrm{p}<0,05)$ entre os animais manejados em potreiros de 20 e $40 \mathrm{~cm}$ de altura de milheto (tabela I), exceto na conformação, onde animais mantidos em pastejo de $20 \mathrm{~cm}$ de altura de milheto apresentaram maior índice de conformação, possivelmente explicado pelo menor gasto de energia que esses animais dispuseram para alimentar-se. Entre as características analisadas o estado de engorduramento da carcaça é o elo para o entendimento entre o que o consumidor deseja de gordura e o que o produtor pode conseguir, através da condição corporal (Osório e Osório, 2005). Segundo, Sañudo e Sierra (1986), o sistema de manejo influencia o estado de engorduramento, principalmente em relação ao tipo de alimento que os animais recebem. No entanto, a diferença na altura de pastejo do milheto não foi suficiente para promover uma mudança no estado de engorduramento dos animais.

Os resultados deste trabalho mostram que o peso corporal foi responsável por 33 \% da conformação corporal (tabela II). Quanto melhor a conformação maior será o rendimento de carcaça e o estado de engorduramento (Charpentier, 1967). Já a compacidade da carcaça mostrou ser um bom estimador da composição regional da carcaça, sendo responsável por 62 e $45 \%$ dos pesos da paleta e perna, respectivamente. Em relação à porção comestível músculo da paleta, a compacidade corporal foi responsável pela variação de 50 \% do músculo desse corte $\left(\mathrm{R}^{2}\right)$, corroborando com resultados de outros autores (Quadros et

Tabela II. Coeficientes de correlação de Pearson de características in vivo e na carcaça. (Pearson correlation coefficients for in vivo and carcass characteristics).

\begin{tabular}{lcccccccccc}
\hline & PC & CC & CFC & CFCA & PCA & PPA & PPE & COM & RC & EE \\
\hline PC & 1,0 & 0,28 & 0,57 & 0,48 & 0,89 & 0,92 & 0,87 & 0,77 & 0,25 & 0,39 \\
CC & 0,28 & 1,0 & 0,46 & 0,45 & 0,28 & 0,41 & 0,36 & 0,10 & 0,14 & 0,33 \\
CFC & 0,57 & 0,46 & 1,0 & 0,42 & 0,49 & 0,54 & 0,58 & 0,30 & 0,10 & 0,22 \\
CFCA & 0,48 & 0,45 & 0,42 & 1,0 & 0,56 & 0,58 & 0,60 & 0,58 & 0,40 & 0,77 \\
PCA & 0,89 & 0,28 & 0,49 & 0,56 & 1,0 & 0,93 & 0,85 & 0,78 & 0,66 & 0,56 \\
PPA & 0,92 & 0,41 & 0,54 & 0,58 & 0,78 & 1,0 & 0,85 & 0,79 & 0,48 & 0,54 \\
PPE & 0,87 & 0,36 & 0,58 & 0,60 & 0,85 & 0,85 & 1,0 & 0,67 & 0,37 & 0,46 \\
COM & 0,77 & 0,10 & 0,30 & 0,58 & 0,78 & 0,79 & 0,67 & 1,0 & 0,37 & 0,54 \\
RC & 0,25 & 0,14 & 0,10 & 0,40 & 0,66 & 0,48 & 0,37 & 0,37 & 1,0 & 0,56 \\
EE & 0,39 & 0,33 & 0,22 & 0,77 & 0,56 & 0,54 & 0,46 & 0,54 & 0,56 & 1,0 \\
\hline
\end{tabular}

$\mathrm{PC}=$ peso corporal; $\mathrm{CC}=$ condição corporal; $\mathrm{CFC}=$ conformação corporal; CFCA= conformação carcaça; $\mathrm{PCA}=$ peso carcaça; $\mathrm{COCA}=$ comprimento da carcaça; $\mathrm{PPA}=$ peso da paleta; $\mathrm{PPE}=$ peso da perna; $\mathrm{COM}=$ compacidade da carcaça; $\mathrm{RC}=$ rendimento carcaça; $\mathrm{EE}=$ estado de engorduramento. 
al., 2007; Pilar, 2002), mostrando a viabilidade da utilização da compacidade corporal como estimador da composição tecidual da paleta.

A conformação da carcaça apresentou alta relação com o estado de engorduramento $\left(\mathrm{R}^{2}=60 \%\right)$. Confirmando resultado de Seideman et al. (1982), os quais afirmam que a conformação está relacionada com a genética e com o estado de engorduramento, já que esta avalia o plano adiposo junto com o muscular em relação ao tamanho do esqueleto que os suportam (Osório e Osório, 2005).

Já com a condição corporal o estado de engorduramento obteve coeficiente de correlação de 0,33 (tabela II). O valor observado neste experimento é inferior aos encontrados por Osório et al. (2004) onde os coeficientes de correlação foram de 0,85 , 0,89 e 0,89 entre a condição corporal, por três avaliadores, com o estado de engorduramento da carcaça, que são superiores ao do presente estudo. Bonacina et al. (2007a) encontraram índices de correlação entre a condição corporal e estado de engorduramento que variaram de 0,65 a 0,89 . Já Jardim et al. (2004) encontraram relação entre a condição corporal e o estado de engorduramento, cujos coeficientes de correlação foram 0,60, 0,51 e 0,73 para três avaliadores. O baixo coeficiente encontrado no presente estudo $(0,33)$ foi devido aos animais estarem bastante uniformes, já que foi realizada uma pré-seleção a campo por condição corporal, o que diminui a variabilidade. A relação entre o estado de engorduramento da carcaça e a condição corporal do animal é importante para avaliar a acuracidade dos avaliadores (Osório e Osório, 2005). A tendência atual é produzir carcaças magras, porém com ótimo grau de gordura, que permita boa apresentação e características sensoriais satisfatórias (Siqueira e Fernandes, 2000).

Não houve diferença entre as características subjetivas de textura e cor, já para o marmoreio houve efeito da altura do milheto $(\mathrm{p}<0,01)$, onde cordeiros que pastejaram em potreiros com $20 \mathrm{~cm}$ de altura de milheto obtiveram maior índice de marmoreio (tabela III). Esse resultado pode ser atribuído ao maior gasto de energia, para alimentar-se, dos animais que estavam em potreiros com milheto na altura de $40 \mathrm{~cm}$. Segundo, Pedroso et al. (2009) plantas mais altas, ou seja, com maior alongamento dos entrenós, faz com que as folhas vivas estejam menos

Tabela III. Médias e erros padrão das características subjetivas (textura, cor e marmoreio), pH e capacidade de retenção de água (CRA) da carne de cordeiros Corriedale manejados em duas alturas de pastejo em milheto. (Means and standard errors of subjective characteristics (texture, color and marbling), $\mathrm{pH}$ and water holding capacity (CRA) of meat from Corriedale lambs grazing two heights of millet).

\begin{tabular}{lccc}
\hline Altura do milheto & $\begin{array}{c}20 \mathrm{~cm} \\
\mathrm{n}=18\end{array}$ & $\begin{array}{c}40 \mathrm{~cm} \\
\mathrm{n}=14\end{array}$ & $\mathrm{p}$ \\
\hline Textura (1-5) & $3,5 \pm 0,16$ & $3,4 \pm 0,18$ & 0,5259 \\
Cor (1-5) & $3,6 \pm 0,11$ & $3,6 \pm 0,12$ & 0,9953 \\
Marmoreio (1-5) & $2,0 \pm 0,10$ & $1,6 \pm 0,11$ & 0,0146 \\
CRA lombo (\%) & $78,38+0,64$ & $77,94+0,72$ & 0,6587 \\
pH 0 hora & $6,72+0,04$ & $6,74+0,05$ & 0,8737 \\
pH 24 horas & $5,73+0,01$ & $5,71+0,01$ & 0,3493 \\
CRA paleta (\%) & $84,50+0,57$ & $83,85+0,65$ & 0,4598 \\
pH 0 hora & $6,73+0,05$ & $6,74+0,05$ & 0,9523 \\
pH 24 horas & $5,79+0,02$ & $5,85+0,03$ & 0,1608 \\
\hline
\end{tabular}

Archivos de zootecnia vol. 63, núm. 241, p. 166. 
Tabela IV. Médias e erros padrão de cor avaliados na carne de ovinos Corriedale manejados em duas alturas de pastejo em milheto. (Means and standard errors for meat color of Corriedale lams grazing millet to two heights).

\begin{tabular}{lccc}
\hline Altura do milheto & $\begin{array}{c}20 \mathrm{~cm} \\
\mathrm{n}=18\end{array}$ & $\begin{array}{c}40 \mathrm{~cm} \\
\mathrm{n}=14\end{array}$ & $\mathrm{p}$ \\
\hline Cor (ferro hemínico/grama) lombo & $59,84 \pm 2,84$ & $62,77 \pm 3,22$ & 0,5045 \\
$\mathrm{~L}^{*}$ & $41,82 \pm 0,68$ & $42,41 \pm 0,77$ & 0,5718 \\
$\mathrm{a}^{*}$ & $19,85 \pm 0,45$ & $19,29 \pm 0,52$ & 0,4290 \\
$\mathrm{~b}^{*}$ & $4,19 \pm 0,32$ & $4,09 \pm 0,37$ & 0,8376 \\
Cor (ferro hemínico/grama) paleta & $60,76 \pm 1,97$ & $62,21 \pm 2,23$ & 0,6306 \\
$\mathrm{~L}^{*}$ & $47,40 \pm 0,65$ & $47,00 \pm 0,73$ & 0,6871 \\
$\mathrm{a}^{*}$ & $20,38 \pm 0,43$ & $21,61 \pm 0,49$ & 0,0695 \\
$\mathrm{~b}^{*}$ & $5,85 \pm 0,34$ & $5,90 \pm 0,38$ & 0,9279 \\
& & & \\
\hline
\end{tabular}

acessíveis ao pastejo por haver um maior espaçamento entre elas, o que limita a quantidade de foragem coletada por bocado. Dessa forma, para compensar esta menor coleta de forragem por bocado, os animais precisam aumentar a taxa de bocados e o tempo de pastejo, com isso, aumentam o gasto de energia na busca da dieta.

Observou-se que os valores de $\mathrm{pH}$ encontrados (tabela III) estão dentro do intervalo considerado normal (Young et al., 2004). Com isso, observando valores normais de queda do $\mathrm{pH}$ da carne, sugere que outros parâmetros indicadores da qualidade, os quais são influenciados pelo $\mathrm{pH}$, como capacidade de retenção de água, cor e textura, apresentarão bons resultados. Os valores encontrados de capacidade de retenção de água corroboram com os resultados obtidos por Bonacina et al. (2007b). Estando esses dentro de uma amplitude considerada normal para carne ovina, indicando que a carne não apresenta problemas exudativos.

A paleta de cordeiros manejados em potreiros com $40 \mathrm{~cm}$ de altura de milheto apresentou valores superiores de cor pelo ferro hemínico e de quantidade de mioglobina, já que o valor de a* foi superior na carne destes cordeiros, mesmo não apresentando diferença significativa em relação aos animais manejados em potreiro com $20 \mathrm{~cm}$ de altura de milheto (tabela IV). $\mathrm{O}$ resultado sugere que esses animais, mantidos em potreiros com maior altura de milheto, tiveram maior gasto de energia para alimentar-se (Pedroso et al., 2009), corroborando com os valores obtidos de marmoreio.

Os resultados obtidos demonstram que não houve diferença $(p<0,05)$ entre os componentes teciduais da paleta de cordeiros alimentados com milheto em altura de pastejo de 20 ou $40 \mathrm{~cm}$ (tabela V). Os componentes teciduais apresentaram pesos superiores ao encontrado por Costa et al. (1999) para a raça Corriedale, já em relação à raça Texel os pesos dos componentes foram semelhantes aos dos cordeiros deste estudo, no entanto, esses animais foram manejados em campo nativo, com provavelmente menor oferta de forragem que o deste trabalho.

Jardim et al. (2000), trabalhando com três sistemas de alimentação, verificaram que cordeiros de pastagem cultivada apresentaram maior percentagem de gordura na paleta e na perna do que os cordeiros mantidos em confinamento e no campo nativo. Já Castro (2002) também trabalhando com diferentes alturas de pastejo em milheto $(10,20,30$ e 40 cm) não verificou diferença 
Tabela V. Composição tecidual da paleta de cordeiros de acordo com a altura do milheto. (Tissue composition of lamb shoulder according to the height of millet grazed).

\begin{tabular}{lccc}
\hline Altura do milheto & $\begin{array}{c}20 \mathrm{~cm} \\
\mathrm{n}=18\end{array}$ & $\begin{array}{c}40 \mathrm{~cm} \\
\mathrm{n}=14\end{array}$ & $\mathrm{p}$ \\
\hline Peso $(\mathrm{kg})$ & 1,285 & 1,271 & 0,5211 \\
Osso $(\mathrm{kg})$ & 0,272 & 0,265 & 0,5256 \\
Osso (\%) & 21,18 & 20,85 & 0,4691 \\
Músculo (kg) & 0,566 & 0,567 & 0,9711 \\
Músculo (\%) & 44,07 & 44,61 & 0,3346 \\
Gordura (kg) & 0,135 & 0,146 & 0,6758 \\
Gordura (\%) & 10,51 & 11,49 & 0,7880 \\
Gordura subcutânea (kg) & 0,096 & 0,096 & 0,9997 \\
Gordura subcutânea (\%) & 7,47 & 7,55 & 0,9556 \\
Gordura intermuscular (kg) & 0,039 & 0,038 & 0,8247 \\
Gordura intermuscular (\%) & 3,04 & 2,99 & 0,9326 \\
Outros tecidos (kg) & 0,162 & 0,156 & 0,4578 \\
Outros tecidos (\%) & 12,61 & 9,12 & 0,3987 \\
& & &
\end{tabular}

no peso das paletas dos cordeiros manejados entre esses tratamentos.

Não houve efeito da altura da pastagem sobre a relação músculo:osso $(2,23 \pm 0,07$ e $2,36 \pm 0,08$, para 20 e $40 \mathrm{~cm}$ respectivamente) e músculo:gordura $(6,41 \pm 1,21$ e 5,83 $\pm 1,34$, para 20 e $40 \mathrm{~cm}$ respectivamente). Costa et al. (1999), trabalhando com animais da raça Corriedale, manejados em campo nativo e abatidos aos 222 dias de idade, observaram valores similares e superiores aos deste estudo para músculo:osso e músculo:gordura, respectivamente. Com o aumento do peso corporal e da idade ao abate, a relação músculo:gordura tende a diminuir, em razão da maturidade fisiológica de cada tecido ter impulso de desenvolvimento em cada fase de vida do animal (Pinheiro et al., 2007).

Mesmo apresentando diferença na quantidade de oferta de forragem, entre as duas alturas de pastejo, essa não foi suficiente para promover diferença na composição tecidual da paleta. Fato que pode ter ser explicado pelo estado de desenvolvimento e crescimento dos cordeiros no momento do abate e/ou pelo grau de maturidade do milheto (Assis et al., 2011).

Por outro lado, parece importante salientar que a preferência do consumidor, condição corporal entre 2 e 3 , em cordeiros da raça Corriedale, corresponde a uma relação músculo:gordura média de 6 (6,41 e $5,83)$, que corresponde aproximadamente 11 $\%$ de gordura (10,51 \% e $11,49 \%$ ) e $45 \%$ de músculo (44,07\% e 44,61\%). Considerando que a gordura é responsável pelo sabor da carne e a busca da relação músculo:gordura preferida pelo consumidor é o que deve determinar o valor da carcaça (Osório et al., 2012).

\section{CONCLUSÃO}

Não há diferença entre as características da carcaça e instrumentais da carne de cordeiros Corriedale manejados em diferentes alturas de pastejo de milheto, quanto às características subjetivas, a altura do milheto influencia o índice de marmoreio na carne. E não há diferença nos componentes teciduais da paleta de cordeiros da raça Corriedale, manejados em altura de pastagem do milheto de $20 \mathrm{~cm}$ e $40 \mathrm{~cm}$ demonstrando que, dentro das condições do estudo, não há necessidade de manejar cordeiros em altura de milheto de $40 \mathrm{~cm}$. 


\section{AGRADECIMENTOS}

À EMBRAPA CPPSUL-Bagé/RS, à FAPERGS e ao CNPq pelo apoio financeiro

\section{BIBLIOGRAFIA}

Assis, R.L.; Costa, K.A.P; Pires, F.R.; Braz, A.J.B.P; Monteiro, F.P.; Moreira, J.F.M. e Cruvinel, W.S. 2011. Composição bromatológica de genótipos de milheto em função do manejo de corte. Global Sci Technol, 4: 21-27.

Bonacina, M.; Osório, J.C.S.; Osório, M.T.M.; Esteves, R.; Jardim, R.; Mendonça, G. e Oliveira, M.M. 2007a. Otimização da avaliação in vivo e da carcaça em cordeiros. Revista FZVA Uruguaiana, 14: 273-286.

Bonacina, M.; Osório, M.T.; Osório, J.C.; Hashimoto, J.H.; Gonçalves, M.; Pradiée, J. e Mendonça, G. 2007b. Qualidade instrumental da carne de cordeiros terminados em diferentes sistemas de produção. XVI Congresso de liniação Científica e IX Encontro de Pós-Graduação. Anais... Universidade Federal de Pelotas. Pelotas.

Castro, C.R.C. 2002. Relações planta-animal em pastagem de milheto (Pennisetum americanum (I.) Leeke) manejada em diferentes alturas com bovinos. Dissertação (Mestrado em Zootecnia) - Curso de Pós-graduação em Zootecnia. Universidade Federal do Rio Grande do Sul. Porto Alegre. $185 \mathrm{pp}$.

Charpentier, J. 1967. Calidad de la canal y de la carne. C.N.R.Z. Jouy-en-Josas. França.

Costa, J.C.C. da, Osório, J.C.S., Osório, M.T.M., Borba, M.F. e Muniz, E.N. 1999. Composição regional e tecidual em cordeiros não castrados. Rev Bras Agrociênc, 5: 50-53.

Durães, F.O.M.; Magalhães, P.C. e Santos, F.G. 2003. Fisiologia da planta de milheto. Circular Técnica Embrapa Milho e Sorgo. Sete Lagoas.

Hornsey, H.C. 1956. The color of cooked cured pork. I. Estimation of the nitric oxide-haem pigments. J Sci Food Agric, 7: 534-540.

Jardim, R.D.; Osório, J.C.S.; Oliveira, N.M.; Osório, M.T.M. e Jardim, P.O.C. 2000. Características produtivas e comerciais de cordeiros da raça Corriedale criados em distintos sistemas nutricionais. Rev Bras Agrociênc, 6: 239242.

Jardim, R.D.; Osorio, J.C.S.; Osório, M.T.M.; ao experimento, à CAPES pela concessão de bolsas de estudo.

Azeredo, D.M.; Mendonça, G. e Esteves, R. 2004. Correlações entre medidas tomadas in vivo e características da carcaça em ovinos Corriedale. XXXI Congresso Brasileiro de Medicina Veterinária. Anais.. São Luís, MA.

NCSS 7.0. 2005. Statistical System for Windows. User's Guide I, II, III. Kaysville, Utah. 2204 pp.

Oliveira, N.M. de; Osório, J.C.S. e Monteiro, E.M. 1998. Produção de carne em ovinos de cinco genótipos. 4. Composição regional e tecidual. Ciênc. Rural, 28: 125-129.

Oliveira, N.R.M.; Moraes, J.C.F. e Borba, M.F.S. 1995. Alternativas para o incremento da produção ovina no sul do Brasil. CPPSUL-ADTT. Bagé. Documentos, 15. 91 pp.

Oliveira, N.M.; Osório, J.C.S. e Monteiro, E.M. 1998. Produção de carne em ovinos de cinco genótipos. 4. Composição regional e tecidual. Ciênc Rural, 28: 125-129.

Osório, J.C.S.; Correa, F.; Osório, M.T.M.; Jardim, R.; Quadro, J.L.; Mendonça, G.; Esteves, R. e Nunes, N. 2004. Avaliação in vivo e da carcaça em cordeiros. XXXI Congresso Brasileiro de Medicina Veterinária. Anais... São Luís-Ma.

Osório, J.C.S. e Osório, M.T.M. 2005. Produção de carne ovina: Técnicas de avaliação in vivo e na carcaça. $2^{a}$ ed. Universidade Federal de Pelotas. Pelotas. 82 pp.

Osório, J.C.S.; Oliveira, N.M.; Osório, M.T.M.; Jardim, R.D. e Pimentel, M. A. 2002. Produção de carne em cordeiros cruza Border Leicester com ovelhas Corriedale e Ideal. Rev Bras Zootecn, 31: 1469-1480.

Osório, J.C.S.; Osório, M.T.M.; Júnior, F.M.V.; Fernandes, A.R.M.; Seno, L.O.; Ricardo, H.A.; Rossini, F.C.; Junior, M.A.P.O. 2012. Rev Agrarian, 5: 433-443.

Pedroso, C.E.S.; Monks, P.L.; Ferreira, O.G.L.; Tavares, O.M. e Lima, L.S. 2009. Características estruturais de milheto sob pastejo rotativo com diferentes períodos de descanso. Rev Bras Zootecn, 38: 801-808.

Pilar, R.C. 2002. Desempenho, características de 


\section{LEMES, OSÓRIO, OSÓRIO, GONZAGA, MARTINS, ESTEVESE LEHMEN}

carcaça, composição e alometria dos cortes, em cordeiros Merino Australiano e cruza lle de France $x$ Merino Australiano. Tese (Doutorado). Universidade de Lavras. Lavras, MG. 55 pp.

Pinheiro, R.S.B.; Sobrinho, A.G.S; Yamamoto, S.M. e Barbosa, J.C. 2007. Composição tecidual dos cortes da carcaça de ovinos jovens e adultos. Pesq Agropec Bras, 42: 565-571.

Quadros, J.L.G; Osório, J.C.S.; Osório, M.T.M.; Mendonça, G.; Gonçalves, M.; Rota; E.L. e Esteves, R. 2007. Relação entre medidas in vivo e na carcaça em cordeiros Corriedale. Rev FZVA Uruguaiana, 14: 217-230.

Sañudo, C. e Sierra, I. 1986. Calidad de la canal en la especie ovina. Ovino One S.A. Barcelona,
Espanha. pp. 127-153.

Seideman, S.C.; Cross, H.R.; Oltjen, R.R. e Schanbacher, B.D. 1982. Utilization of the intact male for red meat production: a review. J Anim Sci, 55: 826-831.

Sierra, I. 1973. Producción de cordero joven y pesado em la raza. Rasa Argonesa. I.E.P.G.E. $\mathrm{n}^{\circ} 18.28 \mathrm{pp}$.

Siqueira, E.R. e Fernandes, S. 2000. Efeito do genótipo sobre as medidas objetivas e subjetivas da carcaça de cordeiros terminados em confinamento. Rev Bras Zootecn, 29: 306-311. Young, O.A.; Wetb, J. and Hartc, A.L. 2004. A method for early determination of meat ultimate pH. Meat Sci, 66: 493-498. 